\title{
Symptomatic pheochromocytoma with normal urinary catecholamine metabolites
}

\author{
Dimitra Zianni ${ }^{1}$, Marinella Tzanela ${ }^{1}$, Serafim Klimopoulos ${ }^{2}$, N.C. Thalassinos ${ }^{1}$ \\ Department of Endocrinology Diabetes and Metabolism ${ }^{1}$ and $^{2 n d}$ Department of Surgery ${ }^{2}$, Evangelismos Hospital, \\ Athens, Greece
}

\begin{abstract}
A 61-year old female presented with paroxysmal hypertension and a $4.5 \mathrm{~cm}$ left adrenal mass on CT scan. Repeated measurements of 24-hour urinary fractionated metanephrines, total catecholamines and vanillylmandelic acid (VMA) were within normal range. A further scintigraphic study with ${ }^{131}$ I -metaiodobenzylguanidine $\left({ }^{131} \mathrm{I}\right.$-MIBG) revealed selective concentration of the radiotracer, corresponding to the CT mass. After adequate preoperative treatment, successful surgical excision of the tumor was performed and the pathological examination confirmed the diagnosis of a cystic pheochromocytoma with a $2 \mathrm{~cm}$ solid tumor. On reevaluation three months later using ${ }^{131} \mathrm{I}-\mathrm{MIBG}$, no evidence of remaining or recurrent disease was found. The patient, off any antihypertensive medication, reported mild recurrent hypertension and panic attacks that were adequately controlled with antidepressants. This is a rare case of a symptomatic pheochromocytoma without elevated urine catecholamines and metanephrines. According to the literature, plasma free metanephrines would be the ideal test for biochemical detection of the tumor. However, in the event that they are not available and there is a high clinical suspicion for the presence of pheochromocytoma, as in our patient, we suggest performance of a functional nuclear medicine study, such as ${ }^{131} I-$ MIBG, to confirm the clinical diagnosis.
\end{abstract}

Key words: Pheochromocytoma, Catecholamines, Metanephrines, VMA

\section{INTODUCTION}

Pheocromocytomas are chromaffin cell tumors typically arising in the adrenal glands and characterized by excessive production of catecholamines. Although occurring in less than 0.2 percent of patients with hypertension, their presence must be ruled out because

Address correspondence and requests for reprints to: Marinella Tzanela, MD, Department of Endocrinology, Diabetes and Metabolism, Evangelismos Hospital, 45-47 Ipsilantou Str., Athens, 10 676, Greece, Tel: 30-210-7201825, Fax: 30-210-7249476, e-mail: stelmar@ath.forthnet.gr Received 20-01-04, Revised 25-02-04, Accepted 10-03-04 if not diagnosed or if left untreated, they can have devastating consequences ${ }^{1}$. The classic triad of symptoms consists of episodic headache, sweating and tachycardia $^{1,2}$. About half of the patients have paroxysmal hypertension, most of the rest have persistent hypertension and some can be completely asymptomatic. Orthostatic hypotension can be present as well, particularly in epinephrine-secreting tumors ${ }^{3-5}$. Pheochromocytomas can also produce other vasoactive compounds (e.g. neuropeptide Y, calcitonin gene-related peptide, vasoactine intestinal peptide, adrenomedullin, atrial natriuretic peptide) that could affect vascular responsiveness and, consequently, the blood 
pressure $^{3,4}$. Exclusively dopamine-producing tumors are extremely rare. Elevations in plasma or urinary dopamine, however, should arouse suspicion of metastatic disease, although it is not a particularly sensitive or specific marker of malignancy ${ }^{6,7}$.

In a clinically suspected pheochromocytoma, the diagnosis is confirmed by measurements of urinary and plasma catecholamines and their metabolites, followed by radiological evaluation to locate the tumor ${ }^{1-6,8}$. Most patients with clinically evident tumors have clearly abnormal values in any of the commonly used tests. Cases of pheochromocytoma without positive biochemical results have been attributed to periodically secreting tumors, small tumors with rapid turnover rate or nonfunctional tumors. We report a 61-year old female with classic clinical manifestations of pheochromocytoma and a left adrenal mass, negative urinary biochemical tests, but positive ${ }^{131} \mathrm{I}$-MIBG scintigraphy for pheochromocytoma. Pathological examination of the surgically excised tumor confirmed the diagnosis.

\section{CASE REPORT}

A 61-year old female was admitted to our department because of paroxysmal hypertension and a left adrenal mass. Her 15-year history of poorly controlled hypertension (170-180/90-110mmHg) had been exacerbated during the past 12 months by spells in spite of her being on intensive treatment with felodipine/metoprolol and enalapril/hydrochlorothiazide. She also reported orthostatic hypotension and three recent episodes of loss of consciousness. A CT scan of the abdomen revealed a mass of $4.5 \mathrm{~cm}$ at the upper pole of the left kidney (Figure 1), with areas of calcification. Areas of low-attenuation were not detected, but no contrast material was used. Her past medical history included dyslipidemia treated with atorvastatin $10 \mathrm{mg}$ o.d., and depression treated with venlafaxine $50 \mathrm{mg}$ o.d. for the last 12 months. No smoking or alcohol drinking was reported. There was a family history of hypertension and cardiovascular mortality in both parents and her four sisters also suffered from hypertension. Upon admission, all medication was discontinued for the appropriate hormonal testing to be reliably carried out. During the first hospital days the patient experienced repeated episodes of paroxysmal hypertension with headaches, sweating, palpitations and anxiety (without clinically significant ECG abnormalities suggesting ischemia) that were efficiently controlled with nifedipine. On examination between episodes, the patient was weak and anxious and could hardly stand. BMI was $26 \mathrm{~kg} / \mathrm{m}^{2}$ (W: $71 \mathrm{~kg}, \mathrm{H}: 165 \mathrm{~cm}$ ). Skin and conjunctivae were pale with cold, sweaty extremities. Body temperature was normal, BP was 170/ $100 \mathrm{mmHg}$ with postural drop to $120 / 60 \mathrm{mmHg}$ and pulse rate $88 / \mathrm{min}$ and $90 / \mathrm{min}$, respectively. Routine biochemical investigations and full blood count at the
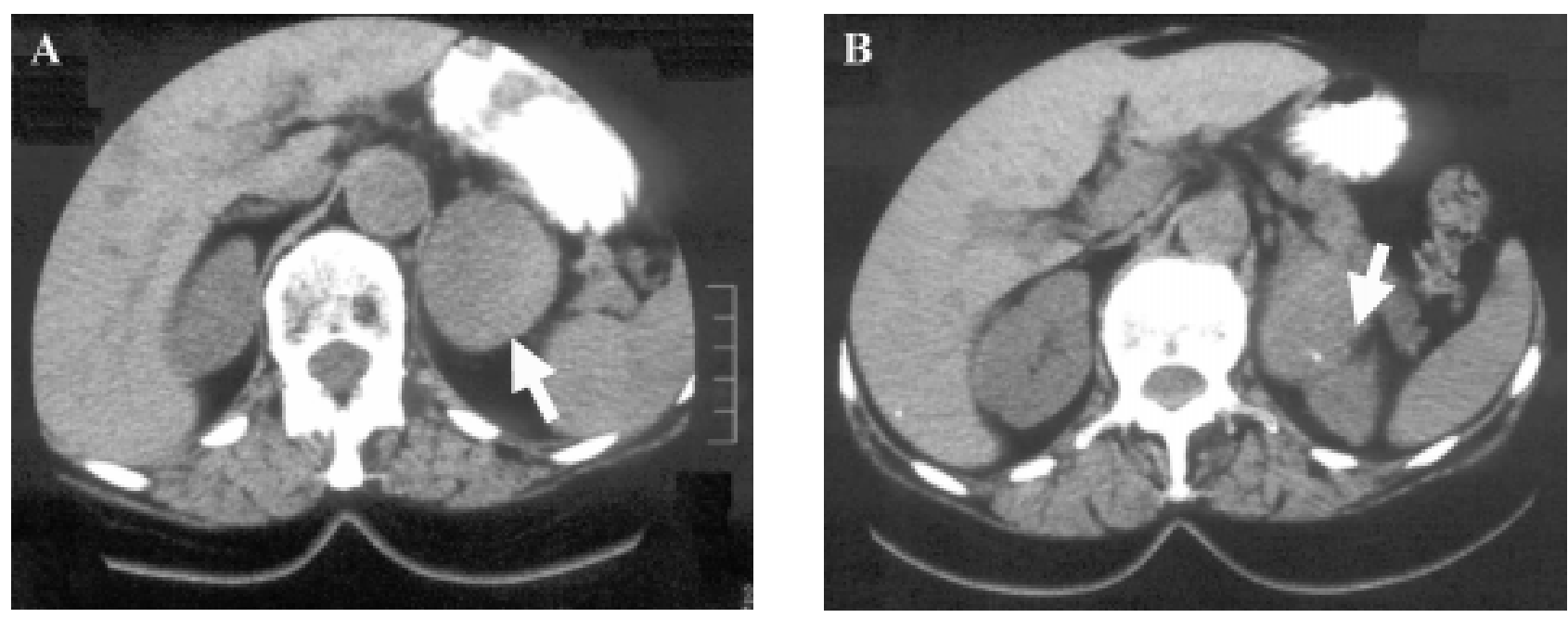

Figure 1. CT of the abdomen demonstrating a mass of $4.5 \mathrm{~cm}$ (arrow 1A) at the upper pole of the left kidney with area of calcification (arrow, 1B). No contrast material was used. No definite abnormalities of the adjacent pancreas, the left and right kidney, the right adrenal or the liver were noted. 
time of presentation revealed normocytic, normochromic anemia, moderately elevated ESR and dyslipidemia. The urine gave a $+/$ - test for protein and hemoglobin. The echocardiogram revealed symmetrical left ventricular hypertrophy, and the ejection fraction was $60 \%$. Specific laboratory investigation included measurement of 24-hour urinary fractionated metanephrines (HPLC), total catecholamines and vanillylmandelic acid (VMA, spectophotometry) on three consecutive days. In addition, twenty-four hour urinary excretion of dopamine and homovanillic acid (HVA, spectophotometry) were measured. All the results were within normal range (Table 1). Plasma catecholamine or metanephrine levels were not determined because pertinent methodology was not available in our institution. Despite the normal levels of urinary catecholamines and their metabolites, suspicion of pheochromocytoma remained very strong on clinical grounds. An imaging study with ${ }^{131} \mathrm{I}$-metaiodobenzylguanidine $\left({ }^{131} \mathrm{I}-\mathrm{MIBG}\right)$ scintigraphy was performed and revealed selective concentration of the radiotracer, corresponding to the CT mass, on the 24to-72-hour images (Figure 2). After adequate adrenergic blockade and hydration, successful surgical excision of the tumor was performed through a transabdominal incision. Immediate dissection of the mass revealed extensive cystic degeneration. Macroscopically, pathological examination revealed a specimen $19.2 \mathrm{gr}$ in weight containing a deep red neoplasm $2 \mathrm{~cm}$ in maximum diameter. Microscopical examination revealed a neoplasm of the left adrenal medulla, with morphologic and immunophenotypic characteristics of pheochromocytoma (Syn + , Chrom,+ S- $100+$ on supportive cells) with central hemorrhagic infiltration. Indications of malignancy (mitosis, necrosis, vascular or capsular infiltration) were not detected. The spec-

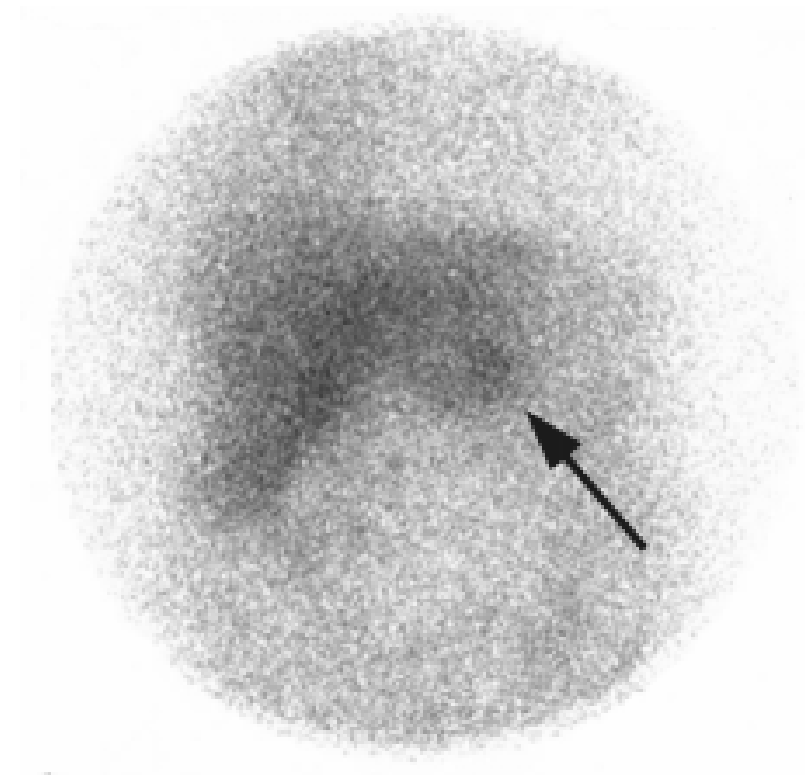

Figure 2. ${ }^{131}$ I-metaiodobenzylguanidine $\left({ }^{131} \mathrm{I}-\mathrm{MIBG}\right)$ scintigraphy demonstrating selective concentration of the radiotracer, anatomically corresponding to the CT mass (arrow), on the 24- to 72-hour images.

imen contained a peripheral rim of adrenal cortex. The patient was discharged a few days later without any antihypertensive medication. She experienced no spells of hypertension and her blood pressure remained normal with no clinically important postural drop. On the follow-up three months later, she had mild recurrent hypertension, asymptomatic orthostatic hypotension and reported panic attacks. Repeated biochemical and imaging studies ${ }^{131}$ I-MIBG scan, thoracic and abdominal MRI) showed no evidence of remaining or recurrent disease. Psychiatric assessment resulted in the diagnosis of persistent depression and the patient was started on appropriate treatment with

Table 1. 24-hour urinary outputs of catecholamines and their metabolites in 3 consecutive collections $\left(\mathrm{V}_{1}, \mathrm{~V}_{2}, \mathrm{~V}_{3}\right)$

\begin{tabular}{lcccc}
\hline & $\mathbf{V}_{\mathbf{1}}: \mathbf{1 8 2 0} \mathbf{m l}$ & $\mathbf{V}_{\mathbf{2}}: \mathbf{2 2 8 0} \mathbf{m l}$ & $\mathbf{V}_{\mathbf{3}}: \mathbf{2 8 8 0} \mathbf{m l}$ & Normal values \\
\hline Total catecholamines & 28 & 39 & 29 & $14-108 \mu \mathrm{g} / 24 \mathrm{~h}$ \\
Fractionated metanephrines & & & & $88-444 \mu \mathrm{g} / 24 \mathrm{~h}$ \\
Normetanephrine & 93 & 343 & 313 & $52-341 \mu \mathrm{g} / 24 \mathrm{~h}$ \\
Metanephrine & 26 & 91 & 89 & $1.8-6.7 \mathrm{mg} / 24 \mathrm{~h}$ \\
VMA & 2,7 & 2.9 & 2.9 & $65-400 \mu \mathrm{g} / 24 \mathrm{~h}$ \\
Dopamine & 126 & 131 & 122 & $<6.2 \mathrm{mg} / 24 \mathrm{~h}$ \\
HVA & 4.1 & 4.5 & 4.4 & \\
\hline
\end{tabular}

VMA: Vanillylmandelic acid, HVA: Homovanillic acidLegends 
good clinical response.

\section{DISCUSSION}

In most cases, the diagnosis of pheochromocytoma is not difficult due to the typical clinical symptoms and signs of catecholamine excess and positive biochemical and imaging results. However, in some patients the biochemical diagnosis and localization of a tumor can be challenging. As presented in this case, a patient with a large mass located in the adrenal gland was found to have negative urine catecholamines and metanephrines. Based on detailed review of the literature, we here present some explanations for such findings ${ }^{6,9-10}$.

The first and rate-limiting step in catecholamines synthesis is conversion of tyrosine to DOPA by tyrosine hydroxylase (Figure 3). DOPA is then converted to dopamine, translocated into storage vesicles, and further converted to norepinephrine. The additional actions of phenylethanolamine $N$-methyltransferase (PNMT), an enzyme mainly localized in chromaffin cells of the adrenal medulla, leads to conversion of norepinephrine to epinephrine. Catecholamines are metabolized by multiple enzymes (Figure 4). Deamination by monoamine oxidase (MAO) to dihydroxyphenylglycol (DHPG) and catechol-O-methyltrans-
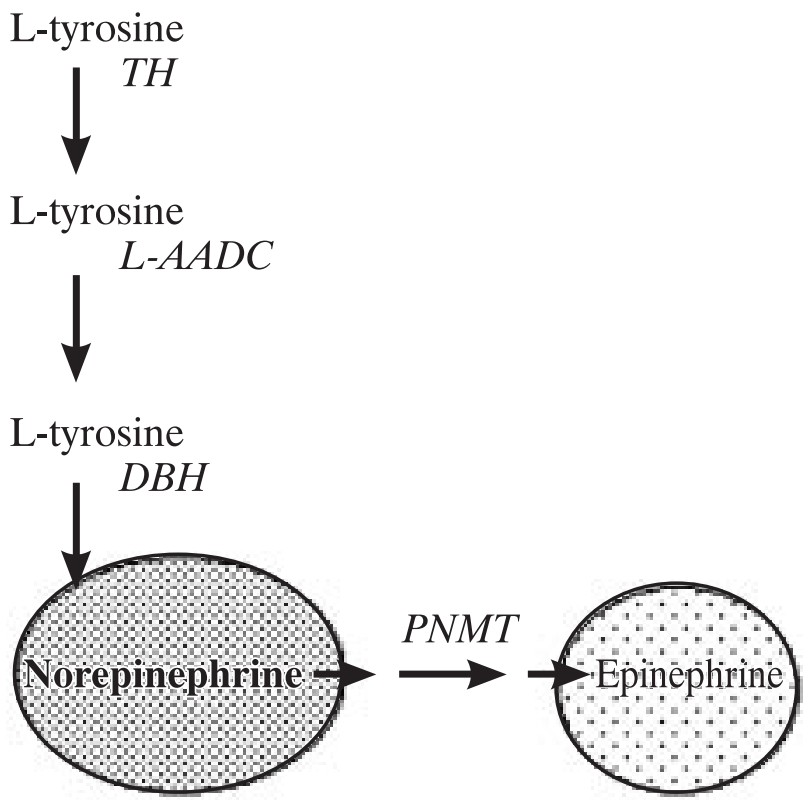

Figure 3. Diagram illustrating the catecholamines biosynthetic pathway in a chromaffin cell. Conversion of L-tyrosine to DOPA takes place in the dopaminergic and noradrenergic neurons of the central nervous system, sympathetic nerves, adrenal and extra-adrenal chromafin cells, and in the gastrointestinal tract and kidney. In chromaffin cells of the adrenal medulla, norepinephrine is converted to epinephrine, both of which are stored in secretory granules. TH, tyrosine hydroxylase; L-AADC, L-aromatic amino acid decarboxylase; $\mathrm{DBH}$, dopamine $\beta$-hydroxylase; PNMT, phenylethanolamine-Nmethyltransferase

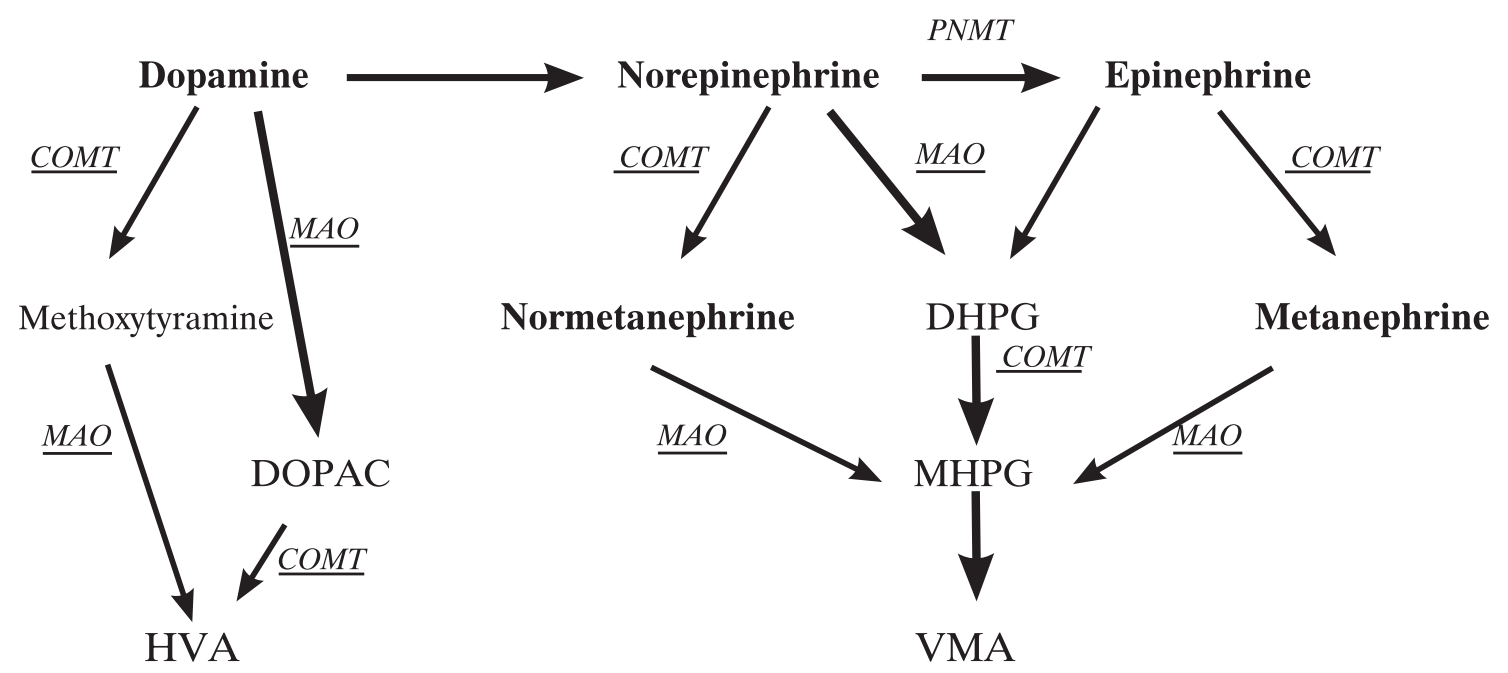

Figure 4. Simplified diagram illustrating catecholamines metabolic pathways. Most of catecholamines metabolism takes place within the same cells where they are synthetized. In sympathetic nerves, MAO is the only metabolizing enzyme. In adrenal medullary chromaffin cells and pheochromocytoma tumor cells, where COMT is prevails, free metanephrines are the main metabolic product. The metabolites produced by MAO can be further metabolized by COMT. The actions of aldose or aldehyde reductase, aldehyde and alcohol dehydrogenaase and the pathways of sulfate conjugation are not shown. DBH, dopamine $\beta$-hydroxylase; PNMT, phenylethanolamine-N-methyltransferase; MAO, monoamine oxidase; COMT, catechol-O-methyltransferase; DHPG, 3,4-dihydroxyphenylglycol. 
ferase (COMT)-catalyzed O-methylation of norepinephrine to normetanephrine, and epinephrine to metanephrine represent the two most important metabolic pathways. With the exception of VMA, which is the principal end product of norepinephrine and epinephrine metabolism in humans, all catecholamines and their metabolites are further converted to sulfate conjugates. The circulatory clearance of catecholamines and their free metabolites is very rapid and their half-lives short, due to active uptake and metabolism throughout the body. In the liver, mainly deaminated metabolites provide the bulk of VMA measurements, while plasma free metanephrines become sulfate-conjugated and return to the bloodstream to be added to the substantial amounts of sulfate-conjugated metabolites derived from the mesenteric organs. They are efficiently handled for elimination by the kidneys and measured by HPLC after a first deconjugation step of acid hydrolysis as a whole (free plus sulfate-conjugated). Thus, they largely reflect levels of sulfate-conjugated metabolites that are produced outside a tumor site. As the circulatory clearance of the end products (sulfate conjugates, VMA) is almost exclusively dependent on renal extraction, it is rather slow and their plasma half-lives relatively long. As a result, plasma concentrations of VMA and sulfate conjugates are much higher than their precursor metabolites and do not accurately reflect relative rates of production. More recent developments include liquid chromatographic measurements of plasma-free metanephrines ${ }^{11-14}$, the single largest source of which are adrenal chromaffin cells. In patients with pheochromocytoma, free metanephrines are produced within tumor cells continuously during the metabolic process and independently of variations in exocytotic catecholamine release caused by sympathoadrenal activation. Although their concentrations in plasma are more than 3 orders of magnitude lower than those measured in the urine, and therefore assays are not easy to establish and run on a routine basis, sensitivity is higher for plasma free metanephrines than for any other test for equivalent levels of specificity ${ }^{15}$. For a patient harboring a pheochromocytoma, a missed diagnosis due to a false-negative result could have serious health consequences. Therefore an appropriately sensitive test should be the first choice for the diagnosis, even if diagnostic specificity is less than ide$\mathrm{al}^{14-17}$. Elimination of false-positive elevations due to medication and use of supplementary tests, such as plasma metanephrines responses to clonidine, can help distinguish true from false-positive results ${ }^{18}$ and reduce costs of the diagnostic workup.

Measurement of urinary excretion of catecholamines and metanephrines has been considered for many years the gold standard for biochemical detection of pheochromocytoma. Urinary fractionated metanephrines seem to be the most useful for exclusion of pheochromocytoma due to high sensitivity $(97 \%)^{15}$. Their specificity is, however, very low. Urinary catecholamines provide good sensitivity as well as high specificity $(99 \%)^{16,17}$. Urinary VMA is mostly useful for confirmation of the diagnosis due to its high specificity $(95 \%)$. The fact that it is present in urine at high concentrations, which makes measurement simple, has made urinary VMA a time-honored, though not necessarily sensitive (63\%), biochemical test ${ }^{15}$. However, 24-hour urine collection may not always be accurate, as e.g. in pediatric patients. Correction of urinary outputs of catecholamines or metanephrines for creatinine excretion can be even more confounding due to dependence of creatinine on diet, muscle mass, physical activity and diurnal variation ${ }^{19}$.

Although in most cases biochemical results are diagnostic, in a small number of patients they can be normal despite the presence of pheochromocytoma. In particular, small tumors are more likely to release unmetabolized catecholamines due to more rapid turnover rate, giving normal values of catecholamine metabolites $^{3}$. Plasma and urinary catecholamines can be normal in episodically secreting tumors, when measured between paroxysmal attacks ${ }^{20}$, also in the presence of so-called "non functional" or "silent" tumors ${ }^{21}$ or, finally, when screening for hereditary tumors. Our patient had been overtly symptomatic during urine collections, thus excluding the possibility of missing a positive test due to episodic secretion. The likelihood of error in the assays applied was virtually eliminated by collecting three consecutive 24-hour urine samples and repeating determination of catecholamines and metanephrines in every sample. Eventually the tumor proved to be cystic with a $2 \mathrm{~cm}$ solid component. Our hypothesis is that released unmetabolized catecholamines or small amounts of metabolic products were not high enough to provide abnormal urinary values, although they were sufficient to provoke the typical symptomatology. It is well known ${ }^{3,4}$ that the severity of clinical symptoms is not correlated with the amount 
of catecholamines released from the tumor. The ${ }^{131} \mathrm{I}-$ metaiodobenzylguanidine (MIBG) scintigraphy, which is a functional, hence highly specific, test (95-100\%) used to locate chromaffin tissue throughout the body, as in multifocal disease or metastases ${ }^{22-25}$, proved positive and pathological examination confirmed the diagnosis.

In conclusion, this case shows that some patients with pheochromocytoma do not have elevated urine catecholamines and metanephrines. As discussed above, the measurement of plasma free metanephrines would be an ideal test for biochemical detection of a tumor but this test is currently available at only a few European institutions. Thus, should plasma free metanephrines not be available and there is a high suspicion for the presence of pheochromocytoma, we suggest the performance of a specific nuclear medicine study, such as ${ }^{131}$ I-MIBG, to detect the tumor.

\section{REFERENCES}

1. Pacak K, Linehan WM, Eisenhofer G, McClellan MW, Goldstein DS, 2001 NIH conference: recent advances in genetics, diagnosis, localization, and treatment of pheochromocytoma. Ann Intern Med 134: 315-329.

2. Bravo EL, Gifford RW Jr, 1984 Current concepts. Pheochromocytoma: diagnosis, localization and management. N Engl J Med 311: 1298-1303.

3. Bravo EL, 1994 Evolving concepts in the pathophysiology, diagnosis, and treatment of pheochromocytoma. Endocr Rev 15: 356-368.

4. Bravo EL, Tagle R, 2003 Pheochromocytoma: State of the Art and Future Prospects. Endocr Rev 24: 539-553.

5. Baxter MA, Hunter P, Thompson GR, London DR, 1992 Phaeochromocytomas as a cause of hypotension. Clin Endocrinol 37: 304-306.

6. Lenz T, Gossmann J, Schulte KL, Salewski L, Geiger H, 2002 Diagnosis of pheochromocytoma 48: 5-18.

7. Yasunari K, Kohno M, Yoshikawa J, 1999 A dopamine secreting pheochromocytoma. Am J Med 106: 599-600.

8. Young Jr WF, 1997 Pheochromocytoma and primary aldosteronism: diagnostic approaches. Endocrinol Metab Clin North Am 26: 801-827.

9. Eisenhofer G, Huynth TT, Hiroi M, Pacak K, 2001 Understanding catecholamine metabolism as a guide to the biochemical diagnosis of pheochromocytoma. Rev Endocrinol Metab Disord 2: 297-311.

10. Axelrod J, 1959 Metabolism of epinephrine and other sympathomimetic amines. Physiol Rev 39: 751-776.

11. Lenders JWM, Keiser HR, Goldstein DS, et al, 1995 Plas- ma metanephrines in the diagnosis of pheochromocytoma. Ann Intern Med 123: 101-109.

12. Raber W, Raffesberg W, Bischof M, et al, 2000 Diagnostic efficacy of unconjugated plasma metanephrines for the detection of pheochromocytoma. Arch Intern Med 160: 2957-2963.

13. Eisenhofer G, 2001 Free or total metanephrines for diagnosis of pheochromocytoma: what is the difference? Clin Chem 47: 988-989.

14. Eisenhofer G, 2003 Editorial: biochemical diagnosis of pheochromocytoma: is it time to switch to plasma-free metanephrines? J Clin Endocrinol Metab 88: 550-552.

15. Lenders JW, Pacak K, Walther MM, et al, 2002 Biochemical diagnosis of pheochromocytoma: which test is best? JAMA 287: 1427-1434.

16. Sawka AM, Jaeschke R, Singh RJ, Young Jr WF, 2003 A comparison of biochemical tests for pheochromocytoma: measurement of fractionated plasma metanephrines compared with the combination of 24-hour urinary metanephrines and catecholamines. J Clin Endocrinol Metab 88: 553-558.

17. Kudva YC, Sawka AM, Young Jr WF, 2003 The laboratory diagnosis of adrenal pheochromocytoma: The Mayo Clinic experience. J Clin Endocrinol Metab 88: 4533-4539.

18. Eisenhofer G, Goldstein D, Walther McCM, et al, 2003 Biochemical diagnosis of Pheochromocytoma: how to distinguish true from false positive test results. J Clin Endocrinol Metab 88: 2656-2666.

19. Weise M, Merke D, Pacak K, et al, 2002 Utility of plasma free metanephrines for detecting childhood pheochromocytoma. J Clin Endocrinol Metabol 87: 1955-1960.

20. Sinclair D, Shenkin A, Lorimer AR, 1991 Normal catecholamine production in a patient with a paroxysmally secreting pheochromocytoma. Ann Clin Biochem 28: 417419.

21. Raber W, Raffesberg W, Kmen E, et al, 2000 Pheochromocytoma with normal urinary and plasma catecholamines but elevated plasma free metanephrines in a patient with adrenal incidentaloma. Endocrinologist 10: 6568.

22. Neumann HP, 2002 Imaging vs. biochemical testing for pheochromocytoma. JAMA 288: 314-315.

23. Sisson JC, Frager MS, Valk TW, et al, 1981 Scintigraphic localization of pheochromocytoma. N Engl J Med 305: 12-17.

24. Graaf JS, Klooster NJJ, Lange WE de, Piers DA, Zwiestra RP, 1991 Comparison of meta-iodo-benzyl-guanidine scintigraphy and computed tomography for localization of pheochromocytoma. Ned Tijdschr Geneesk 135: 2383-2387.

25. Shapiro B, Sisson JC, Shulkin BL, Gross MD, Zempel S, 1995 The current status of meta-iodobenzylguanidine and related agents for the diagnosis of neuro-endocrine tumors. Q J Nucl Med 39: 3-8. 